\title{
Die Aussicht auf EU-Mitgliedschaft und die Stabilisierung Makedoniens
}

\author{
Thorsten Gromes*
}

\begin{abstract}
Macedonia's prospect of accessing the European Union did not prevent the armed conflict between the Albanian National Liberation Army and the state's security forces in 2001. EU representatives, however, contributed to the negotiations, which brought about the so-called Framework Agreement that terminated the violent struggle. The integration prospect encouraged the Albanians not to strive for options beyond Macedonia, while the Macedonians got an incentive to accept the Ohrid Framework Agreement, which they perceive as benefitting the Albanians. Since 2009, the integration process has been blocked by Macedonia's name dispute with Greece. This conflict inhibits the opening of accession negotiations and can worsen the inter-ethnic relations in Macedonia.
\end{abstract}

Keywords: Macedonia, European Union, post-settlement peace-building, inter-ethnic relations Makedonien, Europäische Union, Friedenskonsolidierung, inter-ethnische Beziehungen

A ls 1991 die Scharmützel in Slowenien und 1995 die Kriegshandlungen in Kroatien endeten, spielte die Aussicht, eines Tages der Europäischen Union (EU) beizutreten, keine Rolle. Auch das Dayton-Abkommen für Bosnien und Herzegowina Ende 1995 sah noch keine solche Integrationsperspektive vor. Erst im Zuge der Bemühungen, den Kosovo-Krieg zu beenden, eröffnete die EU 1999 den Nachfolgestaaten Jugoslawiens die Aussicht auf Mitgliedschaft. Als Anfang 2001 die Lage in Makedonien eskalierte, schloss die EU mit der Regierung ein Stabilisierungs- und Assoziierungsabkommen und förderte Verhandlungen, die am 13. August 2001 zum Rahmenabkommen von Ohrid führten und der Gewalt ein Ende setzten. Das Ohrid-Abkommen bekräftigte die Integrationsperspektive und machte die EU zu einem wichtigen Faktor der Friedenskonsolidierung in Makedonien. Dieser Artikel geht der Frage nach, welchen Beitrag die Aussicht auf EU-Beitritt bis Juni 2011 dazu geleistet hat, die inter-ethnischen Beziehungen in Makedonien zu stabilisieren. Wie er zeigt, hat die Aussicht auf Integration sowohl dem Staat Makedonien als auch dem Rahmenabkommen größere Akzeptanz verschafft, die inter-ethnischen Beziehungen entspannt sowie Demokratisierung und Wirtschaftswachstum gefördert. Doch obwohl die EU Fortschritte auf dem Weg zum Beitritt an demokratische Standards knüpfte, blieben Defizite in Sachen Demokratie und Rechtsstaatlichkeit bestehen. Der Namensstreit mit Griechenland blockiert seit Ende 2009 die Aufnahme von Beitrittsverhandlungen und gefährdet die weitere Demokratisierung sowie den inter-ethnischen Ausgleich.

\section{Die EU als Friedensstifter}

Anders als Slowenien, Kroatien, Bosnien und Herzegowina und Kosovo konnte sich Makedonien ohne Krieg oder bewaffneten

\footnotetext{
Dr. Thorsten Gromes, Diplom-Politologe, ist Wissenschaftlicher Mitarbeiter der Hessischen Stiftung Friedens- und Konfliktforschung in Frankfurt am Main. Der Autor dankt der Deutschen Forschungsgemeinschaft für die Förderung des Projekts „Ohne Staat und Nation ist keine Demokratie zu machen“ und den anonymen Gutachtern für konstruktive Kritik.
}

Konflikt von Jugoslawien bzw. Serbien lösen. Auch blieben die innerstaatlichen Beziehungen zwischen Makedoniern und Albanern zumindest vorerst friedlich, und zwar selbst in der Zeit, als im Kosovo-Krieg Hunderttausende Albaner in Makedonien Zuflucht suchten. Darüber hinaus lobten viele Beobachter Makedonien dafür, dass sich in allen Regierungen seit der Unabhängigkeit 1991 makedonische und albanische Parteien die Macht teilten.

Makedonien ist ein multi-ethnisches, multi-linguales und multi-religiöses Land. ${ }^{1}$ In der Volkszählung von 2002 bezeichneten sich 64,2 Prozent der zwei Millionen Bürger als Makedonier, 25,2 Prozent als Albaner, 3,9 Prozent als Türken, 2,7 Prozent als Roma und 1,8 Prozent als Serben (Oschlies 2003: 1). All diese Menschen waren Makedonier im Sinne der Staatsbürgerschaft; fast zwei Drittel identifizierten sich als Makedonier im ethnischen Sinne. Spricht dieser Beitrag im Folgenden von Makedoniern, meint er die größte Ethnie. Die große Mehrheit der Makedonier spricht Makedonisch, schreibt Kyrillisch ${ }^{2}$ und gehört, wie auch die meisten Serben, dem orthodoxen Christentum an. Die Albaner hingegen sprechen Albanisch, benutzen das lateinische Alphabet und die meisten von ihnen teilen mit der Mehrheit der Türken und Roma den muslimischen Glauben.

Die bis 2001 erfolgte Annäherung an die EU konnte nicht verhindern, dass Anfang jenes Jahres ein tödlicher Granatenangriff Makedonien aus seiner vermeintlichen Erfolgsgeschichte riss. Die Gewalt ging von der (albanischen) Nationalen Befreiungsarmee (UÇK) aus, die Militär und Polizeikräfte Makedoniens angriff. Welche Ziele die UÇK ${ }^{3}$ verfolgte, blieb umstritten, auch innerhalb der Albaner. Zunächst hieß es aus der UÇK, sie wolle albanisch besiedelte Gebiete in Makedonien von den „makedonischen Unterdrückern“ befreien und Kosovo oder Albanien angliedern (Ackermann 2001: 119; Naegele 2001; Rozen 2001). Spätestens nach einigen Wochen aber dominierte eine

1 Bei diesem Unterkapitel handelt es sich im Wesentlichen um eine kürzere Version von Gromes 2009b: 4-6. Auch andere Stellen greifen diesen Report auf.

Kyrillische Namen und Texte überträgt dieser Artikel mit diakritischen Zeichen ins Lateinische.

3 Albanische Organisationen kürzt dieser Artikel makedonisch ab, nicht aber die UÇK, die unter dem Namen ONA kaum bekannt ist. 
andere Linie: Die UÇK sah sich in einem Kampf für mehr Rechte der Albaner und griff dabei Forderungen auf, die albanische Parteien schon seit der Unabhängigkeit erhoben hatten. Dabei stellte sie die territoriale Integrität Makedoniens nicht infrage (Daskalovski 2006: 76f.; Rusi 2004).

Zwar konnte die Aussicht auf EU-Beitritt die Eskalation nicht verhindern, dennoch machten sich Repräsentanten der EU darum verdient, den bewaffneten Konflikt beizulegen. Sie und Repräsentanten der NATO bezeichneten die UÇK-Mitglieder zunächst als Terroristen oder kriminelle Extremisten und gaben der Regierung Makedoniens Rückendeckung. Nicht zuletzt aus Solidarität mit der Regierung schloss die EU im April 2001 ein Stabilisierungs- und Assoziierungsabkommen mit Makedonien. Später mahnten EU und NATO die Sicherheitskräfte Makedoniens, sich militärisch zurückzuhalten und drängten erfolgreich auf eine „Regierung der nationalen Rettung“, zusammengesetzt aus den größten makedonischen und albanischen Parteien. Begleitet vor allem von Druck und Hilfe seitens der EU und den USA, handelte diese Regierung das Rahmenabkommen von Ohrid aus (Framework Agreement 2001). Die UÇK nahm auf Wunsch der makedonischen Parteien sowie von EU, NATO und den USA an diesen Verhandlungen nicht direkt teil, ließ sich aber auf das Abkommen ein. ${ }^{4}$ Mit dem Rahmenabkommen endete der bewaffnete Konflikt, der 150-190 Menschen das Leben kostete (Daskalovski 2006: 203; Mitevski 2008: 167).

Das Abkommen zielte vor allem darauf ab, die albanischen Bürger besserzustellen. Viele Albaner hatten sich als Bürger zweiter Klasse empfunden und forderten proportionale Repräsentation in öffentlichen Institutionen, im Parlament einen Konsenszwang für bestimmte Fragen, Albanisch als zweite Amtssprache, höhere Bildung in albanischer Sprache sowie die Dezentralisierung des Staates (Interview mit Ismet Ramadani 2009). Da sie ihre Anliegen missachtet sahen, hatten die meisten albanischen Bürger das Unabhängigkeitsreferendum im September 1991 boykottiert; im Parlament waren die albanischen Abgeordneten der Abstimmung über die Verfassung ferngeblieben (Daskalovski 2006: 64-68, 193). Das Ohrid-Abkommen lässt sich auf die Formel „mehr Rechte für Frieden“ bringen. Es setzte sich zum Ziel, Makedonien näher an die EU und NATO heranzuführen. Politisch motivierte Gewalt wies es bedingungslos zurück. Das Abkommen bekräftigte, es gebe keine territorialen Lösungen für ethnische Fragen. Die UÇK sollte sich entwaffnen und auflösen, eine NATO-Truppe den Waffenstillstand und die Entwaffnung gewährleisten. Im Gegenzug sollten die Ethnien in lokalen und zentralen Institutionen gleichwertig vertreten sein. Das Parlament musste fortan Gesetze mit einem direkten Bezug zu Kultur, Sprache und Symbolen, Bildung, persönlichen Dokumenten sowie lokaler Selbstverwaltung mit einer doppelten Mehrheit verabschieden. Solche Gesetze verlangten eine Mehrheit unter allen Abgeordneten und zusätzlich die Zustimmung der Mehrheit der nicht-makedonischen Abgeordneten. Jede Sprache, die mindestens 20 Prozent der Bürger sprechen, sollte neben Makedonisch Amtssprache werden. Des Weiteren sah das Rahmenabkommen eine Dezentralisierung vor.

4 Zu den Ereignissen von Beginn der Eskalation bis zum Rahmenabkommen: Mitevski 2008.

\section{Konstruktive Wirkungen der Beitrittsaussicht nach dem Ohrid-Abkommen}

Die Aussicht auf Beitritt zur EU steht in dem Ruf, Wichtiges zur Entspannung von Konflikten zwischen ethnisch definierten Gruppen beitragen zu können (Diez et al. 2006: 572f.). Das Integrationsangebot soll Konflikte um Staatsgrenzen entschärfen, da innerhalb der EU Grenzen eine geringere Rolle spielen (Noutcheva et al. 2004: 25-27). Die Europäische Union will zudem Friedensabkommen größere Akzeptanz verschaffen, indem sie Fortschritte im Integrationsprozess an die Umsetzung des Friedensschlusses knüpft. Des Weiteren nutzt die EU das Instrument der Konditionierung, um in beitrittswilligen Staaten demokratische und rechtsstaatliche Standards durchzusetzen. Das dient insofern der Stabilisierung, als Demokratie und Rechtsstaatlichkeit als Ursachen innerstaatlichen Friedens gelten (Senghaas 1995: 198-203). Darüber hinaus ermöglicht die Aussicht auf Integration prominenten Repräsentanten der EU, bei Konflikten im beitrittswilligen Land als allseits akzeptierte Vermittler aufzutreten. Schließlich sollen Annäherung und Mitgliedschaft die Wirtschaft des Beitrittslandes fördern. Auch dies festigt den Frieden, besteht doch eine umso geringere Wahrscheinlichkeit eines Bürgerkrieges je höher das Pro-KopfEinkommen liegt (Collier et al. 2008).

Konstruktive Effekte der Aussicht auf Integration treten nicht voraussetzungslos ein. Solange die politischen Eliten und die Mehrheit der Bürger in den Nachbürgerkriegsgesellschaften den Beitritt nicht wünschen oder für unerreichbar halten, bleibt das Integrationsangebot trotz Konditionierungstaktik ohne positive Wirkung (Kelley 2004; Vachudova 2005). Die geforderten Standards der Demokratie setzt die Konditionierung nur durch, wenn sich die Adressaten darüber gewiss sind, dass sie die in Aussicht gestellten Belohnungen je nach eigenem Handeln gewährt oder vorenthalten bekommen. Daher muss die EU sowohl ihre Forderungen als auch die daran gebundenen Gegenleistungen glaubwürdig und konsistent präsentieren (Schimmelfennig 2008: 920).

\subsection{Die Albaner akzeptieren Makedonien, die Makedonier das Ohrid-Abkommen}

Die Bürger Makedoniens, egal ob Makedonier oder Albaner, teilen den Wunsch, dass ihr Land der EU beitritt. Zwischen 2002 und 2008 unterstützten stets 90 Prozent oder mehr den Integrationsprozess (IRI 2008: Folie 15). Im Jahr 2010 sagten 82 Prozent, sie würden in einem Referendum für den Beitritt stimmen (Gallup 2010: 14). Auch die relevanten politischen Parteien zeigen sich einig im Ziel der EU-Mitgliedschaft, was sich etwa in einer gemeinsamen „Euro-atlantischen Erklärung“ niederschlug (Gaber-Damjanovska/Jovevska 2004: 13f.). Petar Pop-Arsov, Vorsitzender des Ausschusses für europäische Fragen im Parlament Makedoniens, sah einen regelrechten Wettbewerb unter den Parteien, wer am besten für die Integration arbeite. Die EU-Mitgliedschaft, so Pop-Arsov, würde die interethnischen Beziehungen stabilisieren (Interview). Ermira Mehmeti, Abgeordnete der Demokratischen Union für Integration (DUI) der größten albanischen Partei, meinte: “The integration 
is the only re-assurance Albanians have at the moment, the only guarantee to keep Macedonia as it is. What else can keep together two peoples who do not share a common history of constituting a nation?" (Interview). Arjanit Hoxha, Sprecher der Partei für Neue Demokratie, erklärte, aufgrund der euroatlantischen Integration sähen die Albaner keinen Bedarf, für Optionen jenseits des bestehenden Staates zu streiten. Die europäische Integration könne dazu führen, dass alle Albaner, also auch die in Albanien, Kosovo und Serbien, in derselben politischen Gemeinschaft leben (Interview).

Das Integrationsangebot brachte nicht nur dem gemeinsamen Staat Makedonien mehr Akzeptanz, sondern auch dem Rahmenabkommen, das viele Makedonier als Sieg der Albaner und Belohnung für Gewalt wahrnahmen (Vetterlein 2010: 234). Laut einer Erhebung Ende August 2001 lehnten 51 Prozent der befragten Makedonier die Unterzeichnung des Rahmenabkommens ab. Von den befragten Albanern hingegen befürworteten 78 Prozent die Unterzeichnung (Gaber-Damjanovska/Jovevska 2001: 19). Der damalige Ministerpräsident, Ljupčo Georgievski, sagte wenig später, er glaube nicht an das Abkommen, dennoch sei es zu akzeptieren, da Makedonien unter großem äußeren Druck stehe (Gaber-Damjanovska/Jovevska 2001: 7f.). Offenbar konnte die EU mit dem Integrationsprozess glaubwürdig und konsistent einen so großen Anreiz setzen, dass die meisten makedonischen Politiker das Abkommen unterstützten, obwohl es in ihrer Wählerschaft unpopulär war.

Die EU trat auch als Garantiemacht des Ohrid-Abkommens auf, als sie 2003 von der NATO die Führung der internationalen Friedenstruppe übernahm. Ende desselben Jahres schien ihr eine solche Friedensmission angesichts der stabilen Lage im Land nicht mehr erforderlich. Zu dieser Stabilisierung hat die EU nicht zuletzt beigetragen, indem sie wiederholt verdeutlichte, dass nur die Umsetzung des Ohrid-Abkommens den Weg zum Beitritt ebne (Ilievski/Taleski 2009: 360). An diese Bedingungen geknüpft, konnte die Integrationsaussicht Angriffe auf den Friedensschluss und die Verfassungsordnung abwehren. Eine solche Attacke unternahm Georgievski, damals jedoch nicht mehr Ministerpräsident, mit einer Kolumne in der Tageszeitung Dnevnik (18.4.2003). Er forderte, die Staaten des früheren Jugoslawiens nach ethnischen Gesichtspunkten zu teilen und zwischen Albanern und Makedoniern eine Mauer zu bauen. Vertreter der EU, aber auch der USA, der NATO und der Organisation für Sicherheit und Zusammenarbeit in Europa (OSZE) stellten daraufhin klar, dass das Ohrid-Abkommen der einzige Weg nach vorne sei, während alternative Optionen die euroatlantische Integration blockierten (Dnevnik 22.4.2003).

\subsection{Impulse für die Demokratisierung, Vermitt- lung in Konflikten, Förderung der Wirtschaft}

Indem die EU den Stand der Demokratie beobachtete, Mängel benannte und Schritte zu deren Behebung forderte, gab sie Impulse für wichtige Reformen. Sie hatte zum Beispiel die fehlende Unabhängigkeit der Justiz kritisiert. Entsprechend verabschiedete das Parlament Makedoniens Reformen, die den Einfluss der Parteien auf die Ernennung von Richtern reduzieren sollten (Commission of the European Communities 2006:
9-11). Trotz einiger Fortschritte in den letzten Jahren zeigte sich die EU auch 2010 besorgt über politische Eingriffe in die Arbeit der Justiz (European Commission 2010: 12).

Auf die Frage, ob die demokratischen Institutionen ohne die externen Akteure kollabieren könnten, verwies ein Mitarbeiter der EU in Makedonien auf den Konflikt um eine Enzyklopädie, welche die Makedonische Akademie für Wissenschaft und Kunst im September 2009 vorgestellt hatte. Diese bezeichnete die Albaner mit abwertenden Namen und zählte diese nicht zur alteingesessenen Bevölkerung. Da die Akademie als Sprachrohr der größten makedonischen Partei, der regierenden VMRO$\mathrm{DPMNE}^{5}$, galt, forderten die albanischen Parteien, das Buch vom Markt zu nehmen, und verlangten eine Distanzierung von Ministerpräsident Nikola Gruevski. Als dies ausblieb, ließ sich der frühere Sprecher der UÇK, nun Vorsitzender der DUI, Ali Ahmeti, zur Aussage hinreißen, die Enzyklopädie habe den Waffenstillstand zwischen Makedoniern und Albanern gebrochen. Wenig später entspannte sich der Konflikt jedoch - unter anderem nach einer gemeinsamen Erklärung von Ahmeti und Gruevski (Dnevnik, 19.-24.9.2009, 28.-30.9.2009). Der erwähnte Mitarbeiter der EU erklärte, der Streit um die Enzyklopädie habe Makedonien sehr nah an Ereignisse wie 2001 gebracht. Nur ,internationale Faktoren " hätten Gruevski und Ahmeti versöhnt. Der Kabinettsleiter von Ahmeti mochte die internationale Rolle im Konflikt um die Enzyklopädie nicht bestätigen, sagte aber: "The state would collapse without the external presence. Without the external presence, the Framework Agreement would not be implemented and we would come to the situation like before 2001" (Interview mit Irfan Asani).

Die Bürger und Politiker Makedoniens versprechen sich von der Annäherung an die EU und einem späteren Beitritt ökonomische Impulse. Diese hat es gewiss gegeben, wenn auch das Land mit einem Brutto-Inlandsprodukt von rund 4.550 USDollar pro Kopf ${ }^{6}$ nach europäischen Maßstäben noch immer als arm gelten muss. Obwohl Makedonien in den letzten Jahren etwas aufholen konnte, erreichte es 2009 nur 36 Prozent des Durchschnitts der EU-Länder. ${ }^{7}$ Von 2004 bis 2008 wuchs das Bruttoinlandsprodukt jährlich zwischen 4,1 Prozent und 5,9 Prozent, 2009 hingegen sank es um 0,7 Prozent. ${ }^{8}$

\section{Ungewollte Nebeneffekte und Grenzen des EU-Engagements}

Treten Repräsentanten der EU immer wieder als Vermittler auf, können sie damit den Kontrahenten einen Anreiz geben, Konflikte nicht nur in Regierung und Parlament auszutragen. Damit mindern EU-Vertreter ungewollt die Relevanz demokratischer Institutionen. Schwerer wiegt allerdings, dass das Integrationsangebot nicht immer entspannend auf die inter-ethnischen Be-

5 Innere Makedonische Revolutionäre Organisation - Demokratische Partei für Makedonische Nationale Einheit.

6 S. die Daten des Internationalen Währungsfonds unter: www.imf.org/ external/pubs/ft/weo/2010/02/weodata/index.aspx (16.06.2011). Für 2010 und 2011 wird ein Anstieg auf 4.630 US-\$ bzw. 4.870 US-\$ erwartet.

7 So die Angaben von Eurostat unter: http://epp.eurostat.ec.europa.eu/ statistics_explained/index.php/GDP_per_capita,_consumption_per_capita_ and_comparative_price_levels (16.06.2011)

8 www.investinmacedonia.com/Default.aspx?item=menu\&itemid=696\&them eid=304 (16.06.2011). 
ziehungen wirkt. Meint eine Konfliktpartei, die Forderungen der EU folgten zu sehr der Agenda ihres Kontrahenten, wird sie diese Bedingungen zurückweisen und sich vielleicht sogar vom Ziel des Beitritts distanzieren. Selbst wenn alle Konfliktparteien den Beitritt zur EU wollen, vertiefen sich die Gräben, sobald eine Seite der anderen vorwirft, den Beitrittsprozess zu behindern (vgl. Gromes 2009a: 436). Zudem führt selbst eine glaubwürdige und konsistente Beitrittskonditionierung nicht zum von der EU gewünschten Wandel, wenn sich die Regierung des potenziellen Aufnahmelandes vor zu hohe innenpolitische Kosten gestellt sieht (vgl. Schimmelfennig 2008: 921, 932f.).

\subsection{Die EU-Präsenz als Anreiz zum Boykott des Parlaments}

In Makedonien gelten Boykotte der Parlamentssitzungen als beliebtes Mittel im Parteienstreit. Nach der Parlamentswahl 2006 startete die DUI einen Boykott, weil die VMRO-DPMNE nicht mit ihr, der stärksten albanischen Partei, sondern mit der Demokratischen Partei der Albaner (DPA) die Regierung gebildet hatte. Daraufhin drängten EU-Vertreter gemeinsam mit der US-Botschaft auf einen Ausgleich, was zu einer außerparlamentarischen Vereinbarung zwischen der VMRO-DPMNE und der DUI führte. Letztere beendete ihren Boykott, zog aber nicht in die Regierung ein (Dnevnik, 30.5.2007). Lidija Hristova, Professorin für Politikwissenschaft, kommentierte: "The politicians do not want to fight openly but prefer to blackmail, as they know that international community will pressure the government to compromise" (Interview). In der letzten Wahlperiode 2008-2011 boykottierte die DPA unter anderem ab August 2009 das Parlament, da die neue Regierung, geführt von der VMRO-DPMNE und der DUI, den politischen Willen der Albaner systematisch vernachlässige (Dnevnik, 18.8.2009; European Commission 2010: 7). Auch der Sozialdemokratische Bund Makedoniens, die zweitgrößte makedonische Partei, blieb wiederholt den Parlamentssitzungen fern, zuletzt für mehrere Monate aus Protest gegen das Vorgehen der Behörden gegen regierungskritische Medien (OSCE/ODIHR 2011: 3).

\subsection{Demokratische Standards trotz Konditionie- rungspolitik verletzt}

Weder das Beitrittsangebot noch die Appelle von EU-Repräsentanten oder die Präsenz von Wahlbeobachtern der OSZE konnten lange Zeit verhindern, dass die Parlamentswahlen demokratische Standards verfehlten. 2002 kosteten gewaltsame Zwischenfälle mehreren Menschen das Leben (OSCE/ODIHR 2002); vor der Parlamentswahl 2008 ereigneten sich gerade in albanischen Gebieten zahlreiche gewaltsame Zwischenfälle (OSCE/ODIHR 2008: 1, 10 und 18-24). Der damalige Erweiterungskommissar Olli Rehn sowie Botschafter der EU-Mitgliedstaaten betonten, die Politiker Makedoniens dürften nicht erlauben, dass die Wahlen den Beitrittsprozess gefährden (Dnevnik 28.5.2008). Das ständige Pochen von EU-Repräsentanten auf demokratische Standards mag dazu beigetragen haben, dass die vorgezogene Parlamentswahl im Juni 2011 weit friedlicher und regelkonformer verlief als die Wahl drei Jahre zuvor (OSCE/ ODIHR et al. 2011). Zudem wollten sich die albanischen Parteien wohl nicht mehr vorwerfen lassen, Gewalt und Unregelmäßigkeiten in überwiegend albanischen Gebieten blockierten weitere Fortschritte auf dem Weg zum Beitritt. Mit solchen Zwischenfällen hatten die albanischen Politiker ihre Forderung geschwächt, die Makedonier sollten im Namensstreit mit Griechenland nicht die Zukunft des Landes riskieren.

\subsection{Der Beitrittsprozess und die inter- ethnischen Beziehungen unter der Last des Namensstreits mit Griechenland}

Im April 2008 blockierte Griechenland die Aufnahme Makedoniens in die NATO, und im Dezember 2009 verhinderte die griechische Regierung, dass die EU Makedonien ein Datum für den Beginn von Beitrittsverhandlungen nannte. Die Kommission hatte zuvor Bereitschaft signalisiert, den Beginn von Beitrittsgesprächen zu empfehlen. Der Konflikt zwischen Skopje und Athen beschränkt sich nicht auf den verfassungsmäßigen Namen „Republik Makedonien“, den Griechenland als Angriff auf seine territoriale Integrität versteht. Die griechische Regierung stößt sich ebenso an der Selbstbezeichnung als Makedonier, zumal die VMRO-DPMNE die makedonischen Bürger als Nachfahren der antiken Makedonen darstellt. Auch die Bezeichnung „Makedonisch“ für die gängigste Sprache weist die griechische Regierung zurück. Des Weiteren streiten Makedonien und Griechenland über die Umstände einer Rückkehr jener Menschen, die infolge des Griechischen Bürgerkrieges (1946-1949) ins damalige Jugoslawien gekommen waren. Zudem wirft Makedonien Griechenland die Diskriminierung der Slawen im Norden Griechenlands vor, während die griechische Regierung deren slawische Identität und Benachteiligung bestreitet (Kofos 2010; Vankovska 2010).

Angesichts des Vetos Griechenlands sieht sich Makedonien vor eine Beitrittsbedingung gestellt, die über die einst in Kopenhagen vereinbarten Kriterien hinausgeht. Lenkt die griechische Regierung nicht ein, müssen die Makedonier den Namen ihres Staates und ihrer Sprache sowie ihre Selbstdefinition ändern, so sie weiterhin die Mitgliedschaft in der EU wollen. Keinem anderen Beitrittskandidaten, auch keiner der anderen Nachkriegsgesellschaften in Südosteuropa wurde abverlangt, die eigene Identität aufzugeben. Die Hürde des Namensstreits zu überwinden kann daher der makedonischen Regierung nur zu extrem hohen innenpolitischen Kosten gelingen, falls Athen nicht doch noch nachgibt.

Die makedonische Identität gilt den Makedoniern freilich viel mehr als den Albanern. 69 Prozent der Albaner, aber nur drei Prozent der Makedonier meinen, die Republik sollte der NATO und der EU beitreten, auch wenn dies den Verlust des bisherigen Namens bedeuten würde (GALLUP 2008: 10). Bei einer Umfrage im Juli 2010 gaben 82 Prozent der Makedonier, aber nur 18 Prozent der Albaner dem Namen „Republik Makedonien“ Vorrang vor dem Beitritt zu EU und NATO. ${ }^{9}$ Unter den

9 Siehe den Bericht von Kanal 5 vom 12. Juli 2010 unter: www.time.mk/read/ e7ab0dc328/a0ad9aabd1/index.html (16.06.2011). 
Albanern bestehen Zweifel, ob die Makedonier die Integration tatsächlich wollen. So vermutete Ismet Ramadani, von 1991 bis 2006 Mitglied des Parlaments, die VMRO-DPMNE verfolge eine geheime Agenda jenseits der euro-atlantischen Integration (Interview). Ähnlich äußerten sich der frühere Minister für lokale Selbstverwaltung, Rizvan Sulejmani, und der ehemalige Abgeordnete Mersel Bilalli (Interviews). Bilalli warnte zudem vor den Folgen des Namensstreits: „The main fundament for Macedonia is Euro-Atlantic integration. If this fundament is destroyed, Macedonia has no perspective to exist as a state" (Interview). Arjanit Hoxha, Sprecher der Partei für Neue Demokratie, unkte: "If the integration process is blocked, Albanians would think about other ways of politics" (Interview). Der frühere Innen- und Außenminister, Ljubomir Danailov Frčkoski, befürchtete: "If we stay outside EU and NATO, our democracy will be challenged, soon in the mid-term. Staying outside could produce an irrational radicalization" (Interview).

Auch im Juni 2011 stand der Namensstreit der Aufnahme von Beitrittsgesprächen im Weg. Der blockierte Beitrittsprozess hat zwar keinen totalen Stillstand der Reformen bewirkt, lastete aber weiterhin schwer auf den inter-ethnischen Beziehungen. Bei Gesprächen über die Regierungsbildung soll die im albanischen Lager siegreiche DUI von der geschwächten VMRODPMNE Garantien für eine rasche Beilegung des Konflikts mit Griechenland gefordert haben (Utrinski Vesnik, 15.6.2011). Ein Einlenken der größten makedonischen Partei zeichnete sich jedoch nicht ab. Stattdessen ließ sie in der Hauptstadt eine riesige Reiterstatue errichten, die Alexander den Großen verkörpern soll. Die griechische Regierung verurteilte dies als Provokation und wenig hilfreich im Namensstreit (Dnevnik, 23.6.2011). ${ }^{10}$

\section{Fazit}

Insgesamt hat sich in Makedonien die Aussicht auf einen EUBeitritt positiv ausgewirkt. Sie bestärkte die Albaner darin, den gemeinsamen Staat Makedonien zu akzeptieren und gab den Makedoniern einen Anreiz, sich mit dem ungeliebten Rahmenabkommen abzufinden. Fortschritte auf dem Weg zur Mitgliedschaft machte die EU von der Einhaltung demokratischer Standards abhängig. So konnte sie eine Justizreform anstoßen, Gewalt und Unregelmäßigkeiten bei Parlamentswahlen jedoch lange Zeit nicht verhindern. Das Veto Griechenlands gegen Beitrittsverhandlungen mit der EU droht die Konditionierung der Integration als Instrument der Demokratieförderung ins Leere laufen zu lassen. Zudem gefährdet es den Ausgleich zwischen Makedoniern und Albanern. Die EU könnte versuchen, Griechenland im Namensstreit zu einer konstruktiveren Haltung zu drängen. Doch angesichts der innenpolitischen Turbulenzen im Zuge der Schuldenkrise sähe sich wohl keine Regierung in der Lage, den griechischen Bürgern ein Nachgeben gegenüber Makedonien zuzumuten.

10 Im Wahlkampf hatte der DUI-Vorsitzende Ahmeti den Bau solcher Denkmäler kritisiert (Dnevnik, 3.6.2011).

\section{Literatur}

Ackermann, Alice, 2001: On the Razor's Edge: Macedonia Ten Years after Independence, in: IFSH (Hg.), OSCE Yearbook, Baden-Baden, 117-135.

Collier, Paul/Hoeffler, Anke/Söderbom, Måns, 2008: Post-Conflict Risks, in: Journal of Peace Research, 45 (4), 461-478.

Commission of the European Communities, 2006: Former Yugoslav Republic of Macedonia Progress Report, [COM (2006) 649 final\}, Brüssel.

Daskalovski, Židas, 2006: Walking on the Edge. Consolidating Multiethnic Macedonia 1989-2004, Chapel Hill.

Diez, Thomas/Stetter, Stephan/Albert, Mathias, 2006: The European Union and Border Conflicts: The Transformative Power of Integration, in: International Organization, 60 (Sommer), 563-593.

European Commission, 2010: The Former Yugoslav Republic of Macedonia 2010 Progress Report accompanying the Communication from the Commission to the European Parliament and the Council. Enlargement Strategy and Main Challenges 2010-2011, \{COM(2010) 660\}, Brüssel.

Framework Agreement, 2001, www.vmacedonia.com/crisis/doc/ fagreeme.htm (16.06.2011).

Gaber-Damjanovska, Natasha/Jovevska, Aneta, 2001: Barometer: Political Parties Development in the Republic of Macedonia, Nr. 2, http://library.fes.de/pdf-files/bueros/skopje/05321/ barometer02-2001.pdf (16.06.2011).

Gaber-Damjanovska, Natasha/Jovevska, Aneta, 2004: Barometer: Political and Parties Development in the Republic of Macedonia, Nr. 11, www.fes.org.mk/pdf/BarometerDecember2004.pdf (16.06.2011).

GALLUP, 2008: Balkan Monitor. Insights and Perceptions: Voices of the Balkans, 2008 Analytical Report, www.balkanmonitor.eu/files/BalkanMonitor-2008_Analytical_Report.pdf (16.06.2011).

GALLUP, 2010: Balkan Monitor. Insights and Perceptions: Voices of the Balkans, 2010 Summary of Findings, www.balkanmonitor.eu/files/BalkanMonitor-2010_Summary_of_Findings. pdf (23.06.2011).

Gromes, Thorsten, 2009a: The Prospect of European Integration and Conflict Transformation in Bosnia and Herzegovina, in: Journal of European Integration, 31 (4), 431-447.

Gromes, Thorsten, 2009b: Zwischen Zumutung und Versprechen: Die Demokratie in Makedonien, HSFK-Report 8/2009, Frankfurt am Main.

Ilievski, Zoran/Taleski, Dane, 2009: Was the EU's Role in Conflict Management in Macedonia a Success?, in: Ethnopolitics, 8 (3-4), 355-367.

IRI/USAID, 2008: Macedonia 2008.

Kelley, Judith, 2004: International Actors on the Domestic Scene: Membership Conditionality and Socialization by International Institutions, in: International Organization, 58 (Sommer), 425457. 
Kofos, Evangelos, 2010: The Macedonian Name Controversy. Texts and Commentary, in: Südosteuropa, 58 (3), 414-435.

Mitevski, Mančo, 2008: 2001. Vojna so dve lica, Skopje.

Naegele, Jolyon, 2001: UCK Guerrillas Flex Their Muscles, Radio Free Europe/Radio Liberty, Balkan Report, 23.3.2001.

Noutcheva, Gergana/Tocci, Nathalie/Coppieters, Bruno/ Kovziridze, Tamara/Emerson, Michael/Huysseune, Michel, 2004: Europeanization and Secessionist Conflicts: Concepts and Theories, in: Bruno Coppieters/Michael Emerson/Michel Huysseune/Tamara Kovziridze/Gergana Noutcheva/Nathalie Tocci/Marius Vahl, Europeanization and Conflict Resolution. Case Studies from the European Periphery, Gent, 13-62.

Oschlies, Wolf, 2003: Makedonien. Volkszählung und interethnische Turbulenzen, SWP-Diskussionspapier, Berlin.

OSCE/ODIHR, 2002: Former Yugoslav Republic of Macedonia. Parliamentary Elections 15 September 2002. OSCE/ODIHR Election Observation Mission Final Report, Warschau.

OSCE/ODIHR, 2008: The Former Yugoslav Republic of Macedonia: Early Parliamentary Elections 1 June 2008. OSCE/ ODIHR Election Observation Mission Final Report, Warschau.

OSCE/ODIHR/OSCE Parliamentary Assembly/Parliamentary Assembly Council of Europe, 2011: The former Yugoslav Republic of Macedonia: Early Parliamentary Elections, 5 June 2011. Statement of Preliminary Findings and Conclusions, Skopje.

Rozen, Laura, 2001: NLA Autonomy Goal, Institute for War \& Peace Reporting, Balkan Crisis Report 228, 21.3.2001.

Rusi, Iso, 2004: From Army to Party. The Politics of the NLA, in: Institute of War \& Peace Reporting/Defence Academy of the United Kingdom - Conflict Studies Research Centre: The 2001 Conflict in FYROM - Reflections, Surrey, 1-16.

Senghaas, Dieter, 1995: Frieden als Zivilisierungsprojekt, in: ders. (Hg.), Den Frieden denken, Frankfurt am Main 1995, 196-223.

Vachudova, Milada Anna, 2005: Europe Undivided. Democracy, Leverage \& Integration After Communism, Oxford/New York.

Vankovska, Biljana, 2010: David vs. Goliath: The Macedonian Position(s) in the Socalled "Name Dispute" with Greece, in: Südosteuropa, 58 (3), 436-467.

Vetterlein, Merle, 2010: Konfliktregulierung durch powersharing-Modelle: das Fallbeispiel der Republik Makedonien, Baden-Baden.

\section{Interviews}

Anonymes Interview mit einem Mitarbeiter der EU in Makedonien, Skopje, 6.10.2009.

Asani, Irfan („Dreni“), DUI, Kabinettsleiter von Ali Ahmeti, dem Vorsitzenden der DUI, Tetovo, 8.10.2009.

Bilalli, Mersel, Professor an der Juristischen Fakultät der FONUniversität, 1994-2002 Abgeordneter der PDP im Parlament der Republik Makedonien, bei den Ohrid-Verhandlungen Mitglied der PDP-Delegation, Skopje, 7.9.2009.
Danailov Frčkoski, Ljubomir, Professor für Völkerrecht an der Universität St. Kyrill und St. Methodius, ehemaliger Innen- und Außenminister der Republik Makedonien, Skopje, 28.9.2009.

Hoxha, Arjanit, Sprecher der Partei für Neue Demokratie, Skopje, 10.9.2009.

Hristova, Lidija, Professorin für Politikwissenschaft am Institut für Soziologische, Politische und Juridische Forschung, Universität St. Kyrill und St. Methodius, Skopje, 15.9.2009.

Mehmeti, Ermira, DUI, Abgeordnete im Parlament der Republik Makedonien, Skopje, 11.9.2009.

Pop-Arsov, Petar, VMRO-DPMNE, Abgeordneter im Parlament der Republik Makedonien, Vorsitzender des Ausschusses für europäische Fragen, Mitglied im Nationalen Rat für europäische Integration, Skopje, 22.9.2009.

Ramadani, Ismet, 1991-2006 Abgeordneter der PDP im Parlament der Republik Makedonien, bei den Ohrid-Verhandlungen Mitglied der PDP-Delegation, Skopje, 11.9.2009.

Sulejmani, Rizvan, Professor der Universität Tetovo, 19962002 PDP-Abgeordneter im Parlament der Republik Makedonien, früherer Minister für lokale Selbstverwaltung, Skopje, 30.9.2009. 